\title{
Estimated Risks of Fatal Events Associated with Acetaminophen, Ibuprofen, and Naproxen Sodium Used for Analgesia
}

Kenneth J. Rothman* and Lee L. Lanza

RTI Health Solutions, 3040 Cornwallis Road, P.O. Box 12194, Research Triangle Park, NC 27709-2194, USA

\begin{abstract}
Objective: We assessed the net effect on risk of death for within-label use of acetaminophen and the two nonaspirin alternative oral non-prescription analgesics available in the U.S. (ibuprofen and naproxen sodium) in relation to upper gastrointestinal hemorrhage, liver failure, and renal failure.

Methods: For each drug we obtained estimates of recent general-population prevalence of use and the number of deaths in the US from acute liver injury, acute renal failure, and gastrointestinal hemorrhage for the years 2006-2008 in the population age 20 years or older. We searched the literature and reviewed all informative epidemiologic studies to obtain estimates of the relative risks for each analgesic-endpoint combination. From the estimates of prevalence, relative risk and total one-year risk for the U.S. population, we back-calculated the risk among unexposed, using it as a benchmark from which we could obtain the change in absolute risk related to using each analgesic.
\end{abstract}

Results: Under most assumptions for the relative risks among the different analgesics, acetaminophen use carried the smallest absolute increase in risk, the best estimate being about 35 deaths per million in one year. The comparable estimated increased risk of death related to use of ibuprofen or naproxen sodium was 64 deaths for ibuprofen and 118 deaths for naproxen sodium per million person-years respectively.

Conclusions: When non-prescription analgesics are used according to labeled instructions, acetaminophen use appears likely to be associated with smaller combined risks for upper gastrointestinal hemorrhage, liver disease, and renal disease than is use of ibuprofen or naproxen sodium.

Keywords: Analgesics; Non-steroidal anti-inflammatory agents; Ibuprofen; Naproxen sodium; Acetaminophen; Acute liver failure; Acute kidney injury; Gastrointestinal hemorrhage

\section{Introduction}

A considerable proportion of the U.S. population uses nonprescription analgesics on a regular basis [1]. Four oral medications are currently approved in the U.S. for non-prescription analgesia: acetaminophen, aspirin, ibuprofen, and naproxen sodium. As with any pharmaceuticals, these agents carry some risk of adverse events that must be weighed against their therapeutic benefits.

Some cases of acute liver failure have been attributed to nontherapeutic use or unintentional overdose of acetaminophen [2-5]. Acute liver failure is also theoretically possible for those with severely damaged livers who take acetaminophen at recommended doses, although this phenomenon has not been established. Recent recommendations designed to reduce excessive dosing with acetaminophen may also discourage some appropriate use of acetaminophen, diverting patients to use alternative analgesics [6]. These alternatives to acetaminophen carry their own risks and benefits. With nonspecific nonsteroidal anti-inflammatory drugs (NSAIDs), there may be dose-related effects on the gastrointestinal lining and on cardiovascular disease risk [7]. Some of these effects are welcome; for example, aspirin in low doses is recommended to lower risk of myocardial infarction in high risk populations. Other effects, such as acute renal failure and gastrointestinal bleeding, are undesirable.

In this paper, we assess the net effect on mortality risk related to each of the non-aspirin over-the-counter analgesics approved for use in the U.S. There are four major fatal endpoints that are thought to be affected by therapeutic analgesic use. These endpoints are upper gastrointestinal hemorrhage, liver failure, renal failure and a sudden cardiovascular event (either stroke or myocardial infarction). The first three categories of risk convey much smaller risks than cardiovascular events. As a result, any attempt to weigh risks among alternative analgesics would be dominated by the cardiovascular effects of the analgesics. Although acetaminophen does not appear to have any association with cardiovascular mortality, alternative analgesics have effects that may be either protective or causative. Aspirin is used for chemoprevention of cardiac outcomes, although optimal cardiopreventive dosing is too low to offer effective analgesic relief. In contrast, other NSAIDs have been reported to increase cardiovascular mortality [8-10]; two of them, rofecoxib and valdecoxib, were withdrawn in 2004 and 2005 respectively because of an association with cardiovascular deaths $[11,12]$. Because cardiovascular deaths far outnumber deaths from gastrointestinal hemorrhage, liver failure or renal failure, even modest variations in relative risk for cardiovascular deaths would dominate any effects related to deaths from other endpoints.

Nevertheless, for someone who was already getting optimal chemoprevention against cardiovascular death, there remains the question of what the attendant risks might be for someone weighing the use of acetaminophen or an alternative analgesic. Thus, a patient taking $81 \mathrm{mg} /$ day of aspirin to lower cardiovascular risk might still face

*Corresponding author: Kenneth J. Rothman, DrPH, RTI Health Solutions, 3040 Cornwallis Road, P.O. Box 12194, Research Triangle Park, NC 27709-2194, USA, E-mail: krothman@rti.org

Received December 01, 2012; Accepted January 03, 2013; Published January 05, 2013

Citation: Rothman KJ, Lanza LL (2013) Estimated Risks of Fatal Events Associated with Acetaminophen, Ibuprofen, and Naproxen Sodium Used for Analgesia. Adv Pharmacoepidem Drug Safety 2:0124. doi:10.4172/2167-1052.1000124

Copyright: (c) 2013 Rothman KJ, et al. This is an open-access article distributed under the terms of the Creative Commons Attribution License, which permits unrestricted use, distribution, and reproduction in any medium, provided the original author and source are credited. 
the question of what non-prescription analgesic to use for pain. That hypothetical patient could benefit from understanding the comparative risks among the choices available. In this paper we compare the risks for fatal outcomes caused by gastrointestinal hemorrhage, acute liver failure or acute renal failure. We estimated risks for each of these three outcomes for each of the non-aspirin non-prescription analgesics approved in the U.S., in order to estimate the risk of death from these causes related to use of each of these agents.

\section{Methods}

We studied the three oral non-aspirin drugs that are approved for nonprescription analgesia in the US: acetaminophen, ibuprofen and naproxen sodium. For each drug we obtained estimates of the recent general-population point prevalence of use from the most recently available data in the Slone surveys [13,14], which were conducted during the period 2004 to 2007. The Slone Survey inquired about analgesic use among adults age 18+ during the week before the survey. For simplicity we assumed that users reporting that they used one analgesic were nonusers of the others during the reporting period.

We obtained the number of deaths in the U.S. from acute liver injury, acute renal failure, and gastrointestinal hemorrhage for the years 2006, 2007, and 2008 from the National Center for Health Statistics Compressed Mortality File (CMF) [15]. The CMF uses counts of deaths by cause from vital statistics registries and U.S. population estimates from the Bureau of the Census. We restricted the mortality data to age 20 years or older. Types of underlying cause of death were defined by International Classification of Diseases, Tenth Revision (ICD-10) codes [16]. Upper gastrointestinal hemorrhage was defined by codes for gastrointestinal ulcer with hemorrhage or perforation (K25 through K29 range, K92.2). Acute liver failure deaths were defined as codes K72.0 (acute and subacute hepatic failure) and K76.7 (hepatorenal syndrome). For acute renal failure we used code N17, which included acute renal failure with tubular necrosis, or acute cortical necrosis, or other or unspecified pathology. We converted the three years of death data to one-year risks by dividing the average annual deaths in each outcome category over the three years by the average U.S. adult population age $20+$ for those years (Table 1 ).

The total population one-year risk for each endpoint is a weighted average of the risks among nonusers of analgesics and users of each of the three analgesics. Most of the available literature on the risks associated with non-prescription analgesic use compares the risk among users with that of nonusers of that analgesic, and reports results as relative risks. We searched the literature to obtain estimates of the relative risks for each drug-endpoint combination. When more than one study reported findings for a particular drug-endpoint combination, we combined the results in a fixed-effects meta-analysis. For many combinations, there was only one study reporting on the relation. We relied on populationbased or general practice studies whenever available, rather than clinical trials and studies with comparison groups based on either no

\begin{tabular}{|l|l|l|}
\hline Underlying Cause of Death & Total Deaths & One Year Risk (cases/ 100,000) \\
\hline Acute Liver Failure & 2,228 & 0.339 \\
\hline Acute Renal Failure & 24,323 & 3.696 \\
\hline Upper Gastrointestinal Hemorrhage & $26,801^{\mathrm{a}}$ & $4.072^{\mathrm{b}}$ \\
\hline
\end{tabular}

aThe average number of deaths per year in 2006-2008 with the study condition as underlying cause of death was 743 for ALF, 8,108 for ARF, and 8,934 for UGIH

${ }^{\mathrm{b}}$ Average risk of death from the listed underlying cause in a single year during 2006-2008.

Table 1: Number of Deaths and One-Year Risks by Cause Among US Adults Age $20+$ in 2006 through 2008.
NSAID exposure or past exposure. We used three estimates for each of the 9 relative risks that we sought to estimate: we chose a central estimate for our primary evaluation, and we also used a low estimate and a high estimate for sensitivity analyses. Our estimates were based on use of each product according to the label. We did not attempt to incorporate risks stemming from accidental or deliberate overdoses, although when low-dose or high-dose estimates were reported, we used the high-dose result if it was within therapeutic guidelines. The central estimate was either the only reported value or the result of a meta-analysis of reported values. For the low and high estimates we used the lower and upper confidence limits of the reported estimate that we used for the central estimate, or the lower and upper confidence limits of the meta-analysis, unless the lower bound was less than unity, in which case we used unity instead.

For each relative risk, the nonusers could be defined either as nonusers of that product or as nonusers of any analgesic. For our calculations we considered nonusers to be those not using any analgesic. If users of other analgesics are part of the referent group for the relative risks, the estimates for the relative risk obtained may differ from what would have been obtained with just nonusers of analgesics as the referent group. Generally the inclusion of users of other products in the referent group for a specific product would lead to slight underestimates of the relative risk for that product compared with nonusers of any analgesic, presuming that the analgesics elevate risks above the levels in nonusers.

From the relative risk values taken from the literature, we calculated estimates of the absolute risk of each of the fatal endpoints for users of each type of analgesic. We started from the overall risk of each endpoint based on the deaths obtained from the National Center for Health Statistics. That total risk for an endpoint is a weighted average of the risks experienced by each category of analgesic user and the risk among those unexposed to any of the analgesics. From the relative risks and total risk, we back-calculated the risk among unexposed, from which we could obtain the absolute risk for each analgesic category. This calculation assumes that confounding between estimates is comparatively unimportant.

\section{Results}

The prevalence of use of the three analgesics of interest among people 18 years or older was $18 \%$ for acetaminophen [13], $17 \%$ for ibuprofen, and $4.7 \%$ for naproxen sodium [14]. The one-year overall risks of death are given in table 1 for the three endpoints. Risks are low for all three categories, but about one order of magnitude lower for acute liver failure than for acute renal failure or gastrointestinal hemorrhage.

Table 2 gives the range of risk ratio estimates that we assembled from the available literature. The first row indicates what we considered the central estimate for each measure, based on either a fixed effects meta-analysis or the use of a single study where only one was available. The next two rows indicate lower and upper values that we took as the plausible range. These values were either taken from the confidence limits from the single study or meta-analysis, unless additional information on dose or duration of use was available. The underlying literature and reported values are summarized in table 3 , which reports values for any use of the NSAID, for low-dose use, and for high-dose use. These categories are sometimes not available and, when they were, not consistently defined across studies. In table 4 , the number of deaths predicted in a population experience of $1,000,000$ person-years is shown under three different scenarios, using the best estimates for the risk ratios, the low estimates, and the high estimates. 


\begin{tabular}{|l|l|l|l|l|}
\hline & RR Estimate & Acetamin. & Ibuprofen & Naproxen \\
\hline Acute Liver Failure & Best & 1.0 & 2.2 & 2.0 \\
\cline { 2 - 5 } & Low & 1.0 & 1.8 & 1.0 \\
\cline { 2 - 5 } Acute Renal Failure & High & 3.4 & 2.8 & 4.1 \\
\hline & Best & 2.0 & 2.2 & 1.8 \\
\cline { 2 - 5 } & Low & 1.4 & 1.9 & 1.6 \\
\cline { 2 - 5 } & High & 3.0 & 2.5 & 2.0 \\
\hline Upper GI hemorrhage & Best & 1.3 & 2.0 & 4.2 \\
\cline { 2 - 5 } & Low & 1.0 & 1.6 & 3.4 \\
\cline { 2 - 5 } & High & 6.5 & 2.5 & 5.3 \\
\hline
\end{tabular}

Table 2: Range of Relative Risk Estimates for Three Nonprescription Analgesics in Relation to Three Fatal Outcomes.

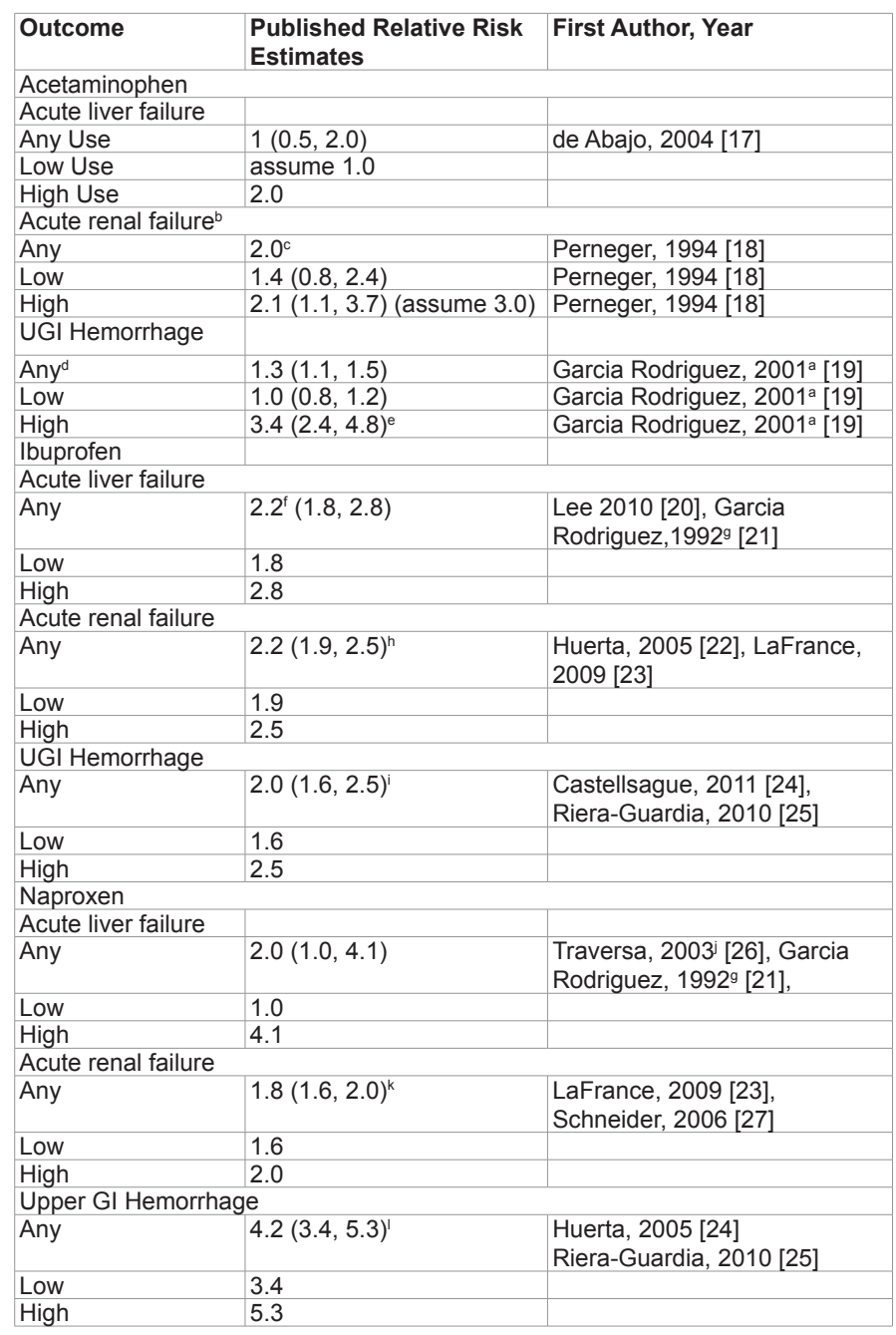

${ }^{a}$ Figures within parentheses are reported $95 \%$ confidence intervals.

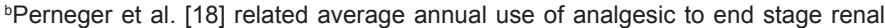
disease.

'Perneger et al. [18] did not report relative risk for overall use; this value is within the range reported for low and high dose.

${ }^{\mathrm{d}}$ Current use.

eEffect for highest recommended daily dose.

fFrom meta-analysis of studies by Lee et al. [20] and Garcia Rodriguez et al. [21].

9Did not separate ibuprofen and naproxen from other NSAIDs.

hFrom meta-analysis of studies by Huerta et al. [22] and LaFrance and Miller [23].

'From meta-analysis of studies by Huerta et al. [24] and Riera-Guardia et al. [25].

iBased on only one exposed event.

${ }^{k}$ From meta-analysis of studies by LaFrance and Miller [23] and Schneider et al. [27].

'From meta-analysis of studies by Huerta et al. [22] and Riera-Guardia et al. [25].

Table 3: Published estimates of risk ratios for three nonprescription analgesics in relation to three fatal outcomes.
Under the best estimate scenario, use of any of the nonprescription analgesics is related to some increase in the overall risk of death from one of the three considered endpoints, compared with nonexposed. Acetaminophen use was estimated to carry the smallest increase in risk, about 35 deaths per million person-years of experience, most of the increase being attributed to an increase in risk of acute renal failure. Increased risk from using ibuprofen was estimated to be nearly twice as great as the increase for acetaminophen, at about 64 deaths per million person-years of experience, with a slightly larger increase in risk than acetaminophen for acute renal failure and a much greater risk for upper gastrointestinal hemorrhage. For naproxen sodium, the increase in risk for acute renal failure is smaller than the increase for either of the other two analgesics, but it is overshadowed by a much greater increase in risk for fatal upper gastrointestinal hemorrhage than the alternative drugs. The overall estimated increase in deaths related to naproxen sodium is 118 per million person-years. From the perspective of switching from acetaminophen to one of the alternatives, there would be an increase in the risk of a fatal adverse event in one of the three categories equal to about 29 deaths per million person-years if the switch is from acetaminophen to ibuprofen and 83 deaths per million person-years if the switch is from acetaminophen to naproxen sodium.

For the scenario based on using all the low estimates, switching from acetaminophen to ibuprofen would result in an estimated increase of 37 deaths per million person-years; for the high estimates, we estimated nearly the same number of deaths from using acetaminophen and ibuprofen. For naproxen sodium, low estimates resulted in an estimate of an increase of 86 deaths per million person-years for those switching from acetaminophen to naproxen, and 53 deaths per million personyears for the high estimates [17-27].

The risks for acute liver failure are so low from any of these drugs that this endpoint barely influences the calculations. Thus, if instead of a RR of 1.0 between acetaminophen and fatal acute liver failure, we had used a RR of 50, the risk of death among acetaminophen users from any of the three endpoints would have been 107 per million personyears instead of 93 per million person-years. The component due to acute liver failure increases from 2.7 to 16.8 deaths per million personyears, with the risk among unexposed changing from 2.7 deaths per million person-years to 0.34 deaths per million person-years. Under this scenario, the risks related to use of ibuprofen and naproxen sodium would be only slightly reduced from the values of 122 per million person-years and 177 per million person-years respectively, and there would be only a small change in the overall risk difference.

The other two endpoints, acute renal failure and upper gastrointestinal hemorrhage, are approximately equally common,

\begin{tabular}{|l|l|l|l|l|l|}
\hline \multicolumn{2}{|c|}{} & Unexposed & Acetamin & lbuprofen & Naproxen \\
\hline Scenario 1 & Acute Liver Failure & 2.7 & 2.7 & 6.0 & 5.4 \\
\hline Best Estimate & Acute Renal Failure & 26.0 & 52.0 & 57.2 & 46.8 \\
\cline { 2 - 6 } & Upper GI Hemorrhage & 29.6 & 38.5 & 59.3 & 124.4 \\
\cline { 2 - 6 } & Total & 58.3 & 93.2 & 122.4 & 176.7 \\
\hline Scenario 2 & Acute Liver Failure & 2.1 & 4.2 & 5.8 & 8.5 \\
\hline All High RRs & Acute Renal Failure & 22.2 & 66.7 & 55.6 & 44.5 \\
\cline { 2 - 6 } & Upper GI hemorrhage & 21.6 & 73.3 & 53.9 & 114.3 \\
\cline { 2 - 6 } & Total & 45.6 & 114.2 & 115.3 & 167.2 \\
\hline Scenario 3 & Acute Liver Failure & 3.0 & 3.0 & 5.4 & 3.0 \\
\hline All Low RRs & Acute Renal Failure & 29.5 & 41.3 & 56.0 & 47.2 \\
\cline { 2 - 6 } & Upper GI hemorrhage & 33.5 & 33.5 & 53.6 & 114.0 \\
\cline { 2 - 6 } & Total & 66.0 & 77.8 & 115.0 & 164.1 \\
\hline
\end{tabular}

Table 4: Predicted Deaths per Million Person-Years by Cause of Death and Analgesic for Three Scenarios. 
but the estimates from the literature of increased risk for each of the analgesics are more narrowly spread for acute renal failure than for upper gastrointestinal hemorrhage. Thus, the assumptions about the magnitude of effects for upper gastrointestinal hemorrhage dominate the risk calculations and projected differences in risk among these analgesics.

\section{Discussion}

This analysis is premised on an array of reported findings from the literature on analgesics, some of which are subject to considerable error. We also made a number of assumptions for ease of comparison. We assumed that all analgesics would be taken according to label directions. Thus, our estimate that acetaminophen likely does not increase risk of fatal liver failure is intended to describe the relation when users follow the instructions on the label. According to the FDA, "The risk of liver injury primarily occurs when patients take multiple products containing acetaminophen at one time and exceed the current maximum dose of $4000 \mathrm{mg}$ within a 24-hour period" [28]. Nevertheless, even when the risk ratio for liver injury from acetaminophen was assumed to be 50 rather than 1, the overall risk of death from any of these three outcomes studied changed very little, because deaths from liver injury were so rare.

We also assumed that effects of analgesics were uniform over age, sex and other variables, and that there was no uncontrolled confounding that would have influenced the interpretation. Another assumption was that each person uses only one analgesic at a given time, although in reality there could be combination therapy. We ignored non-fatal adverse effects and limited attention among fatal outcomes to three endpoints, liver disease, renal disease, and upper gastrointestinal hemorrhage. Doing so involved what was perhaps the most important assumption, namely that the cardiovascular effects were constant across all the analgesics, and that all users were receiving optimal cardioprotective doses of aspirin. For someone who was not taking low-dose aspirin, the higher-dose of aspirin taken for analgesia might lower cardiovascular risk as a secondary effect and thus offset some of the increase in risk from upper gastrointestinal bleeding. But if the same person already takes low-dose aspirin, thus reducing the risk of cardiovascular death but not achieving analgesia, acetaminophen appears likely to be associated with a lower risk of death than ibuprofen or naproxen sodium, the other two non-aspirin non-prescription alternatives on the U.S. market.

\section{Acknowledgement}

This work was supported by a contract between McNeil Consumer Healthcare and RTI-Health Solutions. RTI Health Solutions is an independent, non-profit research organization that does work for government agencies and pharmaceutical companies. No ethical review was needed for this work.

\section{Support}

This work was supported by a contract between McNeil Consumer Healthcare and Rti-Health Solutions. Rti health solutions is an independent, non-profit research organization that does work for government agencies and pharmaceutical companies.

\section{References}

1. Kaufman DW, Kelly JP, Rosenberg L, Anderson TE, Mitchell AA (2002) Recent patterns of medication use in the ambulatory adult population of the United States: the Slone survey. JAMA 287: 337-344.

2. Beer C, Pakravan N, Hudson M, Smith LT, Simpson K, et al. (2007) Liver unit admission following paracetamol overdose with concentrations below current UK treatment thresholds. QJM 100: 93-96.
3. Dart RC, Bailey E (2007) Does therapeutic use of acetaminophen cause acute liver failure? Pharmacotherapy 27: 1219-1230.

4. Larson AM, Polson J, Fontana RJ, Davern TJ, Lalani E, et al. (2005) Acetaminophen-induced acute liver failure: results of a United States multicenter, prospective study. Hepatology 42: 1364-1372.

5. Norris W, Paredes AH, Lewis JH (2008) Drug-induced liver injury in 2007. Curr Opin Gastroenterol 24: 287-297.

6. Food and Drug Administration (2009) Department of Health and Human Services: Organ-specific warnings; internal analgesic, antipyretic and antirheumatic drug products for over-the-counter human use; FR DOC \# E99684. Fed Regist. 2009; 74: 19385-19409.

7. Conaghan PG (2012) A turbulent decade for NSAIDs: update on current concepts of classification, epidemiology, comparative efficacy, and toxicity. Rheumatol Int 32: 1491-1502.

8. García Rodríguez LA, Varas-Lorenzo C, Maguire A, González-Pérez A (2004) Nonsteroidal antiinflammatory drugs and the risk of myocardial infarction in the general population. Circulation 109: 3000-3006.

9. Ray WA, Stein CM, Hall K, Daugherty JR, Griffin MR (2002) Non-steroidal antiinflammatory drugs and risk of serious coronary heart disease: an observational cohort study. Lancet 359: 118-123.

10. Varas-Lorenzo C, Castellsague J, Stang MR, Perez-Gutthann S, Aguado J, et al. (2009) The use of selective cyclooxygenase- 2 inhibitors and the risk of acute myocardial infarction in Saskatchewan, Canada. Pharmacoepidemiol Drug Saf 18: 1016-1025.

11. http://www.merck.com/news/newsredirect.html

12. McDaid C, Maund E, Rice S, Wright K, Jenkins B, et al. (2010) Paracetamol and selective and non-selective non-steroidal anti-inflammatory drugs (NSAIDs) for the reduction of morphine-related side effects after major surgery: a systematic review. Health Technol Assess 14: 1-153, iii-iv.

13. Slone Epidemiology Unit, Boston University School of Public Health: Acetaminophen use in the adult population of the United States in 20042007. Results of a population-based telephone survey (unpublished report for McNeil). May, 2009.

14. (2006) Slone Epidemiology Center at Boston University: Patterns of medication use in the United States. A report from the Slone Survey.

15. Compressed Mortality File 1999-2008. CDC WONDER On-line Database November, 2010. The current release for years $1999-2008$ is compiled from Compressed Mortality File 1999-2008 Series 20 No. 2N, 2011 on March 27, 2012.

16. World Health Organization. International Statistical Classification of Diseases and Related Health Problems. 10th Revision. Version for 2006.

17. de Abajo FJ, Montero D, Madurga M, Garcia Rodriguez LA (2004) Acute and clinically relevant drug-induced liver injury: a population-based case-control study. Br J Clin Pharmacol 58: 71-80.

18. Perneger TV, Whelton PK, Klag MJ (1994) Risk of kidney failure associated with the use of acetaminophen, aspirin, and nonsteroidal antiinflammatory drugs. N Engl J Med 331: 1675-1679.

19. García Rodríguez LA, Hernández-Díaz S (2001) Relative risk of upper gastrointestinal complications among users of acetaminophen and nonsteroidal anti-inflammatory drugs. Epidemiology 12: 570-576.

20. Lee CH, Wang JD, Chen PC (2010) Increased risk of hospitalization for acute hepatitis in patients with previous exposure to NSAIDs. Pharmacoepidemio Drug Saf 19: 708-714.

21. García Rodríguez LA, Pérez Gutthann S, Walker AM, Lueck L (1992) The role of non-steroidal anti-inflammatory drugs in acute liver injury. BMJ 305: 865-868.

22. Huerta C, Castellsague J, Varas-Lorenzo C, García Rodríguez LA (2005) Nonsteroidal anti-inflammatory drugs and risk of ARF in the general population. Am J Kidney Dis 45: 531-539.

23. Lafrance JP, Miller DR (2009) Selective and non-selective non-steroidal antiinflammatory drugs and the risk of acute kidney injury. Pharmacoepidemiol Drug Saf 18: 923-931.

24. Castellsague J, Pisa F, Rosolen V, Riera-Guiardia N, Giangreco M, et al. (2011) 
Citation: Rothman KJ, Lanza LL (2013) Estimated Risks of Fatal Events Associated with Acetaminophen, Ibuprofen, and Naproxen Sodium Used for Analgesia. Adv Pharmacoepidem Drug Safety 2:0124. doi:10.4172/2167-1052.1000124

Page 5 of 5

Use of nimesulide and other NSAIDs and the risk of upper gastrointestinal complications in Firuli Venezia Giulia, Italy. Analysis of cases with codes with high positive predictive value. Abstract 409. PDS 20: S178.

25. Riera-Guardia N, Castellsague J, Calingaert B, Varas-Lorenzo C, FourrierRéglat A, et al. (2010) Nonsteroidal anti-inflammatory drugs and upper gastrointestinal complications. Meta-analysis of epidemiological studies. Poster. International Conference on Pharmacoepidemiology.

26. Traversa G, Bianchi C, Da Cas R, Abraha I, Menniti-Ippolito F, et al. (2003)
Cohort study of hepatotoxicity associated with nimesulide and other nonsteroidal anti-inflammatory drugs. BMJ 327: 18-22.

27. Schneider V, Lévesque LE, Zhang B, Hutchinson T, Brophy JM (2006) Association of selective and conventional nonsteroidal antiinflammatory drugs with acute renal failure: A population-based, nested case-control analysis. Am J Epidemiol 164: 881-889.

28. Food and Drug Administration. Department of Health and Human Services. Questions and Answers about Oral Prescription Acetaminophen Products to be Limited to 325 mg Per Dosage Unit Updated Aug 1, 2011 